\title{
USP33 is a Biomarker of Disease Recurrence in Papillary Thyroid Carcinoma
}

\author{
Meng Jia ${ }^{\mathrm{a}}$ Yaman Guo ${ }^{\mathrm{b}} \quad$ Xiubo $\mathrm{Lu}^{\mathrm{a}}$ \\ aDepartment of Thyroid Surgery, The First Affiliated Hospital of Zhengzhou University, Zhengzhou, \\ Henan, 'Department of Endocrinology, Henan Provincial People's Hospital, Zhengzhou, Henan, People's \\ Republic of China
}

\section{Key Words}

Papillary thyroid carcinoma $•$ Prognosis $・$ Robo1 • USP33

\begin{abstract}
Background/Aims: To investigate the clinical significance and functional mechanisms of deubiquitinase USP33 in papillary thyroid carcinoma (PTC). Methods: Immunohistochemistry staining was conducted to explore the expression of USP33 in PTC tissues and adjacent normal thyroid tissues. Patients' prognosis was evaluated by disease-free survival. The prognostic role of USP33 was tested by univariate and multivariate analyses. To confirm the effect of USP33 in cell proliferation and invasion, overexpression and knockdown of USP33 were performed in two PTC cell lines. Besides, cell cycle, immunoprecipitation, and apoptosis experiments were conducted to further explore the signaling pathways. Results: By analyzing series of 158 PTC tissues, we found that USP33 was down-regulated in tumor tissue compared with normal thyroid tissues, which was closely associated with lymph node metastasis $(P<0.001)$. In particular, univariate and multivariate analyses indicated that USP33 was an independent prognostic biomarker for PTC, low USP33 expression indicated high recurrence risk. Cellular studies with TPC-1 and BCPAP cells demonstrated that USP33 can attenuate the cell capacities of proliferation, migration and invasion. Fluorescenceactivated cell sorting experiment found no significant effect of USP33 on cell cycle, whereas the apoptotic caspase proteins were activated by USP33-overexpression. Moreover, the interaction between USP33 and Robo1 protein was identified, and knockdown of Robo1 enhancing the oncogenic effect upon USP33-knockdown, suggesting that USP33 may inhibit tumor progression through Robo1 signaling. Conclusions: Our data demonstrated that USP33 downregulation in PTC tissues was correlated with poor clinical outcome, which may serve as a novel biomarker and potential therapeutic target.

\section{Introduction}

Thyroid cancer is the most prevalent endocrine malignancy, comprising $3 \%$ of all newlydiagnosed human cancers [1]. Papillary thyroid cancer (PTC) is the major histological type 


\section{Cellular Physiology Cell Physiol Biochem 2018;45:2044-2053 \\ and Biochemistry Published online: March 12, $2018 \quad \begin{aligned} & \text { DO 2018 The Author(s). Published by S. Karger AG, Basel } \\ & \text { www.karger.com/cpb }\end{aligned}$ \\ Jia et al.: USP33 is a Tumor Suppressor in PTC}

of thyroid cancer with the incidence rising worldwide [2]. Despite the good prognosis (10 year overall survival $>90 \%$ ) for most PTC patients [3], cervical lymph node metastasis and early invasion has a high occurrence [4]. Till now, the prognosis of PTC has been reported to depend on several well-established clinicopathologic parameters including age, tumor size, histologic subtype, extrathyroidal extension (ETE), and lymph node metastases [3, 5]. Generally, 10-15\% of PTC patients are diagnosed with distant metastases and poor clinical outcomes [6]. In addition, disease recurrence exists in 5-20\% of all patients even underwent total thyroidectomy [7]. Therefore, further investigation for invasion regulators will lead to a better understanding of the invasive process, promising identification of novel prognostic biomarkers, and potential drug development for metastatic PTC.

Besides protein expression, the post-translational modifications (PTMs) of proteins have been recognized to play important roles in tumor development during the past decades, with p53 as a good example [8,9]. Among numerous PTMs, ubiquitination is nonnegligible in modulating protein activity and degradation, which is drawing attentions in oncology studies $[10,11]$. Ubiquitination balance is regulated by ubiquitin ligases and deubiquitinases (DUBs), dysregulation of either one may lead to physiopathologic disorders including neoplasm. Increasing evidence has verified the prognostic and therapeutic roles of DUBs [12]. DUBs refer to a large family of proteases that can cleave the peptide between ubiquitin and its substrate protein, thus reversing the degradation effects by ubiquitin ligases [13]. DUBs can be classified into two classes: cysteine proteases and metalloproteases. The cysteine proteases include ubiquitin-specific proteases (USPs), ubiquitin C-terminal hydrolases (UCHs), Machado-Josephin domain proteases (MJDs) and ovarian tumor proteases (OTU); whereas the metalloprotease contains only the Jab1/ Mov34/Mpr1 Pad1 N-terminal+ (MPN+) (JAMM) domain proteases [14].

Recently, several ubiquitin ligases (e.g. ZNRF3) and DUBs (e.g. USP22) have been indicated to regulate the tumor development of PTC $[15,16]$. However, the detailed roles of ubiquitin ligases and DUBs are completely different due to their specific catalyzing substrates. In the current study, we tested the mRNA and protein levels of USP33 in PTC tissues and adjacent thyroid tissues for the first time. Clinical data implicated USP33 as an independent prognostic factor for the disease-free survival (DFS) of PTC patients. In addition, we found that Robo1 can enhance the tumor suppressing effects of USP33 on PTC cells, indicating their potential interaction and signaling cross-talk.

\section{Materials and Methods}

\section{Patient and samples}

The case study included 158 PTC patients underwent surgical resection in The First Affiliated Hospital of Zhengzhou University between 2008 to 2014. Freshisolated PTC tissues and matched adjacent normal thyroid tissues were obtained from the Department of Thyroid Surgery, including 2 males and 7 females who underwent total thyroidectomy for PTC in our hospital $(n=9)$. Tissue samples were paraffin embedded or frozen in liquid nitrogen and stored at $-80^{\circ}$ C. None of the patients received any pre-operation drug treatment. The patients were staged according to the current TNM classification system, and underwent post-operative radioactive iodine ablation (RIA) according to American Thyroid Association Guidelines [17]. Retrospectively collected clinical information included gender, age, tumor size, ATA risk, TCI, ETE, histological type, lymph node status and recurrence. Complete follow-up details were obtained in all PTC patients and were included for DFS analysis.

\section{Immunohistochemistry (IHC) and IHC evaluation}

Specimens were paraffin-embedded and cut into $4 \mu \mathrm{m}$ serial sections, deparaffinized, blocked, and incubated at $4^{\circ} \mathrm{C}$ overnight with the primary antibodies, followed by horseradish peroxidase-labeled secondary antibody. Immunoreactivity was detected with avidin-biotin-HRP complex (Thermo Fisher Scientific, Pittsburgh, PA, USA). Negative controls were performed by using PBS instead of primary antibody. The primary antibodies used for IHC in this study included mouse monoclonal antibody to USP33 (Santa 


\section{Cellular Physiology Cell Physiol Biochem 2018;45:2044-2053 \\ \begin{tabular}{l|l|l} 
and Biochemistry 10.1159/000488041 & $\begin{array}{l}\text { C } 2018 \text { The Author(s). Published by S. Karger AG, Basel } \\
\text { www.karger.com/cpb }\end{array}$
\end{tabular}}

Jia et al.: USP33 is a Tumor Suppressor in PTC

Cruz Biotechnology, \#sc100632) and rabbit polyclonal antibody to Robo1 (Santa Cruz Biotechnology, \#sc25672).

The expression of USP33 or Robo1 was analyzed by both staining intensity and percentage of positive stained cells. In brief, five randomized visual fields were observed and photographed. Scores representing the numbers of cells stained (range score) were graded according to the percent of positively stained cells: $<25 \%$ (score 0 ), $25-50 \%$ (score 1), and $>50 \%$ (score 2). Scores representing the intensity of staining were " 0 " (unstained, negative), "1" (brown stained, weakly positive), or " 2 " (dark brown stained, strongly positive). The final expression score was obtained by multiplying the two scores above, ranging $0-4$. Only score 2 or 4 was grouped into high expression, while score $<2$ were classified as low expression.

Quantitative real-time reverse transcription polymerase chain reaction ( $R T-q P C R$ )

Total RNA was isolated from 9 fresh-frozen PTC tissues and 9 paired adjacent normal thyroid tissues using TRIzol reagent (Invitrogen, Carlsbad, CA, USA). Reverse transcription was carried out using a Goscript Reverse Transcription System Kit (Promega, Madison, WI, USA) according to the manufacturer's instructions. The primers for USP33 and the internal control ( $\beta$-actin) were designed as following:

USP33 forward primer: CAGTCTGCATCTCCAAAGAGAA;

USP33 reverse primer: ACTACTGGACCCCAAAACCA;

$\beta$-actin forward primer: ATAGCACAGCCTGGATAGCAACGTAC;

$\beta$-actin reverse primer: CACCTTCTACAATGAGCTGCGTGTG.

Quantitative PCR (qPCR) was performed using the Real-Time PCR detection system (Applied Biosystems, Shanghai, China) with $2 \times$ SYBR Green II/ROX qPCR Master Mix (Takara Bio Inc.). Relative mRNA expression was calculated using the delta threshold cycle $(\Delta \Delta \mathrm{Ct})$ method and normalized to $\beta$-actin expression. The experiments were repeated three times independently.

Western Blot and co-immunoprecipitation (CoIP)

Cells or tissues were lysed in RIPA buffer supplemented with protease and phosphatase inhibitors (Roche, Ventana, AZ, USA), and total protein was quantified by the Bradford method (Bio-Rad Laboratories, Hercules, CA, USA). For Western blot analysis, equal amounts of protein ( $30 \mu \mathrm{g})$ were separated by $10 \%$ SDSPAGE and blotted onto PVDF membranes (Bio-Rad Laboratories). The membranes were then incubated with $5 \% \mathrm{BSA}$ for $45 \mathrm{~min}$ at room temperature and then with primary antibodies at $4^{\circ} \mathrm{C}$ overnight, followed by incubation with the secondary antibodies conjugated to HRP (Abcam, Cambridge, MA, USA). Immunoblotting were detected using enhanced chemiluminescence (Thermo Fisher Scientific, Pittsburgh, PA, USA).

For the CoIP experiment, transfected cells were lysed and obtained the supernatant after high speed centrifuge. Cell lysates were incubated overnight at $4^{\circ} \mathrm{C}$ with anti-HA beads or anti-Flag M2 beads (SigmaAldrich), respectively. Immunocomplexes on the beads were washed and eluted in SDS-PAGE sample buffer, heated at $95^{\circ} \mathrm{C}$ for $5 \mathrm{~min}$, followed by the Western Blot procedure above.

\section{Cells and transfection}

Human thyroid papillary carcinoma cell line TPC1 was obtained from the American Type Culture Collection (ATCC, Manassas, VA, USA). BCPAP cell line was purchased from Chinese Academy of Science. TPC- 1 and BCPAP cells were cultured at $37^{\circ} \mathrm{C}$ with $5 \% \mathrm{CO}_{2}$ in DMEM with $10 \%$ fetal bovine serum (FBS) (Life Technologies, Carlsbad, CA, USA), $100 \mu \mathrm{g} / \mathrm{mL}$ penicillin and $100 \mu \mathrm{g} / \mathrm{mL}$ streptomycin.

USP33-pCMV plasmids were obtained from Addgene (\#ABIN3319474), and subcoloned by adding the Flag tag. Robo1-pCMV plasmids were also purchased from Addgene (\#ABIN3379828) and modified by adding an HA tag. Oligomer siRNAs for USP33 were ordered from Ribobio (Guangzhou, Guangdong, China) with the sequence GAUCAUGUGGCGAAGCAUA. All transfections were achived using Lipofectamine 2000 (Invitrogen, Carlsbad, CA, USA) following manufacturer's protocol.

\section{Proliferation, migration and invasion}

The MTT assay were adopted to assess cell proliferation ability. Briefly, 1000 cells in $200 \mu \mathrm{l}$ FBScontaining DMEM were seeded into each well of 96-well plates and cultured for seven days. Three wells from each group were detected every day. For the detection, $100 \mu \mathrm{l}$ fresh medium containing MTT (5 mg/ $\mathrm{ml}$ ) was put into each well and incubated at $37^{\circ} \mathrm{C}$ for 4 hours, then the medium was replaced by $150 \mu \mathrm{l}$ DMSO and shaken at room temperature for $10 \mathrm{mins}$. The absorbance was measured at $490 \mathrm{~nm}$ wavelength. The experiments were repeated three times independently, and results were presented as means $\pm \mathrm{SE}$. 
For migration assay, PTC cells were seeded into six-well plates and cultured until full confluence. Multiple scratch wounds were created in the monolayers, then the cells were cultured routinely. Images were taken at $\times 100$ magnifications after $24 \mathrm{~h}$ to analyze the wound closure. The mean closure of the wound was calculated based on three individual measurements for each wound, and results were presented as means \pm SE.

A 24-well transwell plate with inserted chambers (8- $\mu$ m pore size; Costar, New York, NY, USA) was used to measure the invasion of PTC cells. Chambers were coated with Matrigel (BD Biosciences, East Rutherford, NJ, USA) and cells were then applied to the upper compartment. After cultured for $24 \mathrm{~h}$, invading cells were fixed and stained with $0.1 \%$ crystal violet. Invading cells were imaged and counted in five randomly selected fields under an inverted microscope $(\times 200)$.

\section{Statistics}

Statistical analysis was performed using SPSS version 20.0 (IBM, New York, NY, USA). Fisher exact or Pearson chi-square tests were used to compare the differences between high-USP33 expression group and low-USP33 expression group. Survival curves were plotted using the Kaplan-Meier method and compared by the log-rank test. Significant factors in the univariate models were further assessed by a multivariable Cox regression model. For all tests, $P<0.05$ was considered statistical significant.

Ethics

Written informed consent was obtained from each patient prior to initiation of this study. This study was approved by the Ethical Committee of The First Affiliated Hospital of Zhengzhou University.

\section{Results}

\section{Patients information}

A total of 158 patients with PTC were enrolled on this study (Table 1). Median age of the patients was 47 years (range 16-81). Among them, 121 $(76.6 \%)$ were females and $37(23.4 \%)$ were males. There were 101 (63.9\%) patients with tumor diameter less than $20 \mathrm{~mm}, 57(36.1 \%)$ patients with tumor size larger than $20 \mathrm{~mm}$. The ATA (American Thyroid Association) risk was graded as low risk for $88(55.7 \%)$ patients, middle risk for $64(40.5 \%)$ patients, and the other $6(3.8 \%)$ patients were graded as high ATA risk. Thyroid capsular invasion (TCI) was positive in $54(34.2 \%)$ patients, and extrathyroidal extension (ETE) was positive in $73(46.2 \%)$ patients. Only $20(12.7 \%)$ patients were with follicular histological type, and others were all classic PTC. Lymph node (LN) metastasis was positive in $45(28.5 \%)$ patients, and $33(20.9 \%)$ patients were classified as TNM stage III-IV. The median follow-up was 68 months (range 14-106 months), during which 48 patients $(30.3 \%)$ experienced disease recurrence.
Table 1. Clinicopathologic characteristics of the PTC patients and their correlation with USP33 expression

\begin{tabular}{|c|c|c|c|c|}
\hline \multirow[t]{2}{*}{ Variables } & \multirow{2}{*}{$\begin{array}{c}\text { Cases } \\
(n=158)\end{array}$} & \multicolumn{2}{|c|}{ USP33 expression } & \multirow[t]{2}{*}{$\mathrm{P}$ value } \\
\hline & & Low $(n=58)$ & $\operatorname{High}(n=100)$ & \\
\hline Age (years) & & & & 0.065 \\
\hline$\leq 45$ ys & 72 & 32 & 40 & \\
\hline$>45$ ys & 86 & 26 & 60 & \\
\hline Gender & & & & 0.314 \\
\hline Female & 121 & 47 & 74 & \\
\hline Male & 37 & 11 & 26 & \\
\hline Tumor size & & & & $<0.001^{*}$ \\
\hline$\leq 20 \mathrm{~mm}$ & 101 & 25 & 76 & \\
\hline$>20 \mathrm{~mm}$ & 57 & 33 & 24 & \\
\hline ATA risk & & & & 0.602 \\
\hline Low & 88 & 34 & 54 & \\
\hline Middle & 64 & 21 & 43 & \\
\hline High & 6 & 3 & 3 & \\
\hline TCI & & & & 0.682 \\
\hline Negative & 104 & 37 & 67 & \\
\hline Positive & 54 & 21 & 33 & \\
\hline ETE & & & & 0.289 \\
\hline Negative & 85 & 28 & 57 & \\
\hline Positive & 73 & 30 & 43 & \\
\hline Histological type & & & & $0.623 \S$ \\
\hline Classic & 138 & 52 & 86 & \\
\hline Follicular & 20 & 6 & 14 & \\
\hline LN metastasis & & & & $<0.001^{*}$ \\
\hline Negative & 113 & 30 & 83 & \\
\hline Positive & 45 & 28 & 17 & \\
\hline TNM stage & & & & 0.005 \\
\hline I-II & 125 & 39 & 86 & \\
\hline III-IV & 33 & 19 & 14 & \\
\hline
\end{tabular}




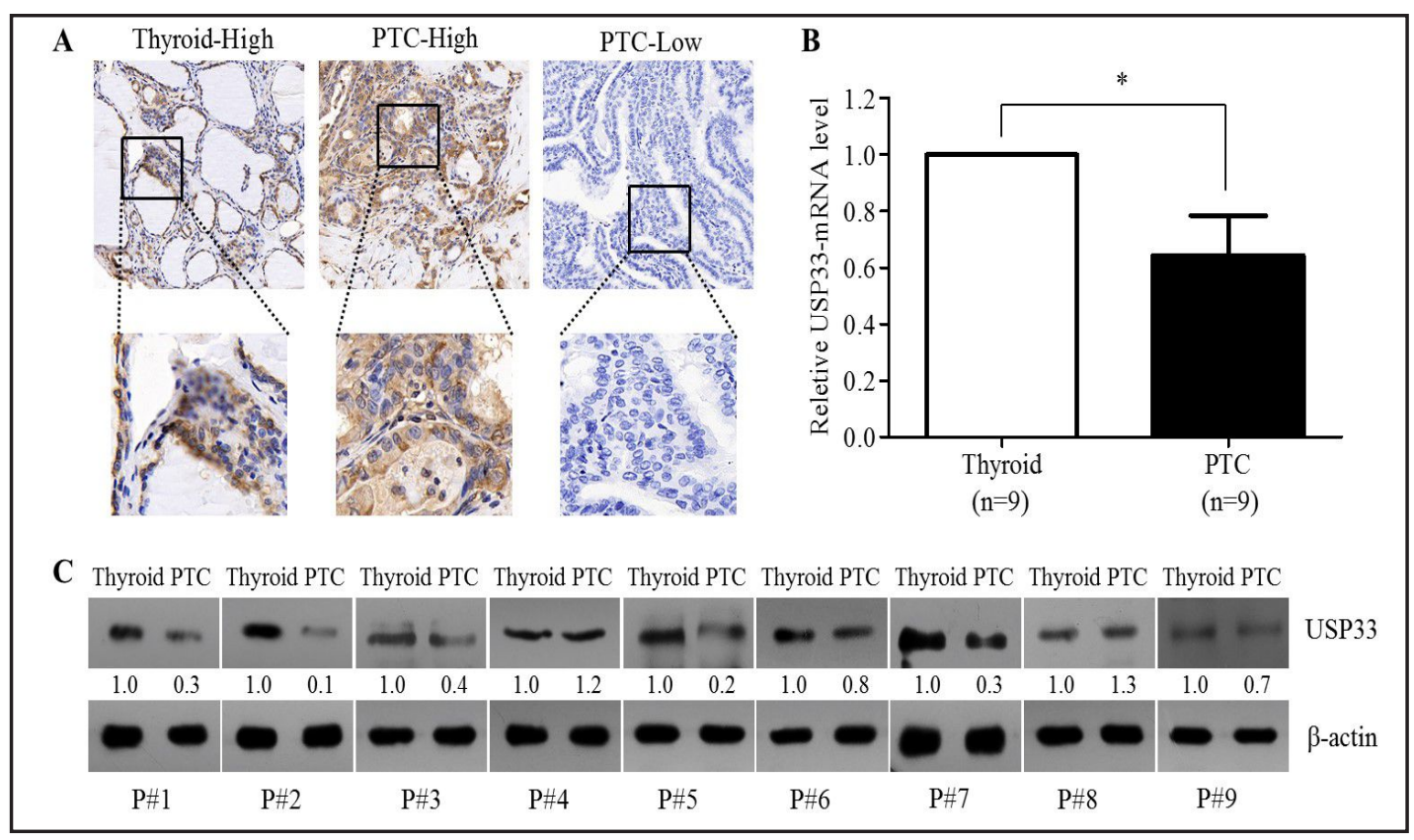

Fig. 1. USP33 is downregulated in PTC tissues. (A) Protein expression levels of USP33 in paraffined thyroid tissues and PTC tissues were measured by IHC, showing the positive immunoreactivity in cytoplasm. (B) The mRNA levels of USP33 from 9 fresh-resected PTC tissues and paired adjacent thyroid tissues were analyzed by RT-qPCR normalized with $\beta$-actin-mRNA level, indicating that USP33 was down-regulated in tumor tissues. (C) The protein extraction from the 9 pairs tissues were tested with USP33 antibody by Western Blot, among which 7 pairs $(7 / 9,77.8 \%)$ showed lower USP33 protein levels in PTC tissues compared with thyroid tissues.

\section{USP33 is downregulated in high risk PTC patients}

USP33 showed different expression patterns among PTC patients, with the major staining located in cytoplasm (Fig. 1A). By testing the fresh-frozen tissues, we found that the mRNA expression levels of USP33 were down-regulated in PTC tissues compared with adjacent normal thyroid tissues (Fig. 1B). Consistently, protein expression levels of USP33 were also lower in tumor tissues than that in normal thyroid tissues (Fig. 1C).

To investigate whether USP33 showed clinical significance in PTC patients, we compared the correlations between USP33 and clinicopathological characteristics (Table 1). It has been observed that patients with larger tumor size or positive LN metastasis were more likely to show decreased USP33 expression $(\mathrm{P}<0.05)$, suggesting that USP33 may show tumorsuppressing roles in PTC. No significant difference was found between USP33 expression levels and other clinicopathological findings.

\section{USP33 is an independent prognostic factor}

To further investigate whether USP33 was correlated with patients' survival, we performed univariate analysis and found that low-USP33 expression patients showed shorter DFS compared with those possessing higher US33 levels (5-year DFS 71.7\% and 93.4\%, respectively). Other prognostic parameters included tumor size, ATA risk, ETE status, LN metastasis and TNM stages (Table 2, Fig. 2).

We then enrolled the parameters above into a multivariate analysis to identify the independence (Table 3). Statistical results revealed that low levels of USP33 were independently correlated with poorer $\mathrm{DFS}(\mathrm{P}=0.020, \mathrm{HR}=2.09)$. Besides, the data indicated that larger tumor size $(\mathrm{P}<0.001, \mathrm{HR}=4.06)$, positive $\mathrm{ETE}(\mathrm{P}=0.009, \mathrm{HR}=2.34)$, positive $\mathrm{LN}$ metastasis $(\mathrm{P}=0.022, \mathrm{HR}=2.19)$ and advanced TNM stages $(\mathrm{P}=0.049, \mathrm{HR}=1.91)$ were all independent prognostic factors for the DFS of PTC patients. 
USP33 inhibits oncogenic characteristics of PTC cells

To investigate the effects of USP33 on tumor biology, we chose two human PTC cell lines, TPC1 and BCPAP cells, respectively, for the in vitro experiments. We transfected cells with USP33 plasmids or siRNA, and found that overexpression of USP33 markedly down-regulated cell viability of the TPC- 1 and BCPAP cells as compared with mock control from $72 \mathrm{~h}$ to 96 h (Fig. 3A). To further identify its antitumor effects were resulted from attenuated cell proliferation or enhanced apoptosis, we next analyzed the cell cycle by using fluorescence-activated cell sorting (FACS) strategy. FACS revealed no statistical difference among the three groups in PTC cells (Fig. 3B). However, USP33 overexpression significantly activated the caspase 9 and caspase 3 proteins (Fig. 3C), indicating its potential role in promoting cell apoptosis.

The effects of USP33 on the migration and invasion of PTC cells were also analyzed using woundhealing and transwell assays, respectively. The results showed that both the migratory and invasive abilities of the PTC cells were

Table 2. Univariate analysis. ${ }^{*} \mathrm{P}<0.05$ was considered as statistically significant. Abbreviations: ATA, American Thyroid Association; ETE, Extrathyroidal extension; LN, Lymph node; TCI, Thyroid capsular invasion; USP33, Ubiquitin specific peptidase 33

\begin{tabular}{|c|c|c|c|c|}
\hline \multirow[t]{2}{*}{ Variables } & \multirow{2}{*}{$\frac{\text { Cases }}{(n=158)}$} & \multicolumn{2}{|c|}{ Disease-free survival } & \multirow[t]{2}{*}{$P$ value } \\
\hline & & Mean \pm SEM (Months) & 5 -year (\%) & \\
\hline Age (years) & & & & 0.242 \\
\hline$\leq 45$ ys & 72 & $90.7 \pm 3.4$ & $89.2 \%$ & \\
\hline$>45$ ys & 86 & $85.9 \pm 2.9$ & $83.8 \%$ & \\
\hline Gender & & & & 0.884 \\
\hline Female & 121 & $87.7 \pm 2.6$ & $86.7 \%$ & \\
\hline Male & 37 & $85.1 \pm 3.6$ & $83.6 \%$ & \\
\hline Tumor size & & & & $<0.001^{*}$ \\
\hline$\leq 20 \mathrm{~mm}$ & 101 & $95.7 \pm 1.9$ & $96.7 \%$ & \\
\hline$>20 \mathrm{~mm}$ & 57 & $69.8 \pm 4.3$ & $64.3 \%$ & \\
\hline ATA risk & & & & $0.006^{*}$ \\
\hline Low & 88 & $87.7 \pm 2.6$ & $89.8 \%$ & \\
\hline Middle & 64 & $91.5 \pm 3.4$ & $85.8 \%$ & \\
\hline High & 6 & $53.5 \pm 14.8$ & $33.3 \%$ & \\
\hline TCI & & & & 0.541 \\
\hline Negative & 104 & $86.7 \pm 2.6$ & $74.1 \%$ & \\
\hline Positive & 54 & $88.8 \pm 3.8$ & $87.6 \%$ & \\
\hline ETE & & & & $0.018^{*}$ \\
\hline Negative & 85 & $93.4 \pm 2.5$ & $92.4 \%$ & \\
\hline Positive & 73 & $81.5 \pm 3.6$ & $78.5 \%$ & \\
\hline Histological type & & & & 0.746 \\
\hline Classic & 138 & $87.6 \pm 2.3$ & $86.4 \%$ & \\
\hline Follicular & 20 & $87.3 \pm 6.6$ & $81.8 \%$ & \\
\hline LN metastasis & & & & $<0.001^{*}$ \\
\hline Negative & 113 & $94.2 \pm 1.9$ & $95.4 \%$ & \\
\hline Positive & 45 & $69.6 \pm 5.2$ & $61.1 \%$ & \\
\hline TNM stage & & & & $<0.001^{*}$ \\
\hline I-II & 125 & $93.6 \pm 2.0$ & $93.2 \%$ & \\
\hline III-IV & 33 & $66.9 \pm 5.9$ & $59.1 \%$ & \\
\hline USP33 expression & & & & $<0.001^{*}$ \\
\hline Low & 58 & $73.0 \pm 4.2$ & $71.7 \%$ & \\
\hline High & 100 & $94.9 \pm 2.1$ & $93.4 \%$ & \\
\hline
\end{tabular}

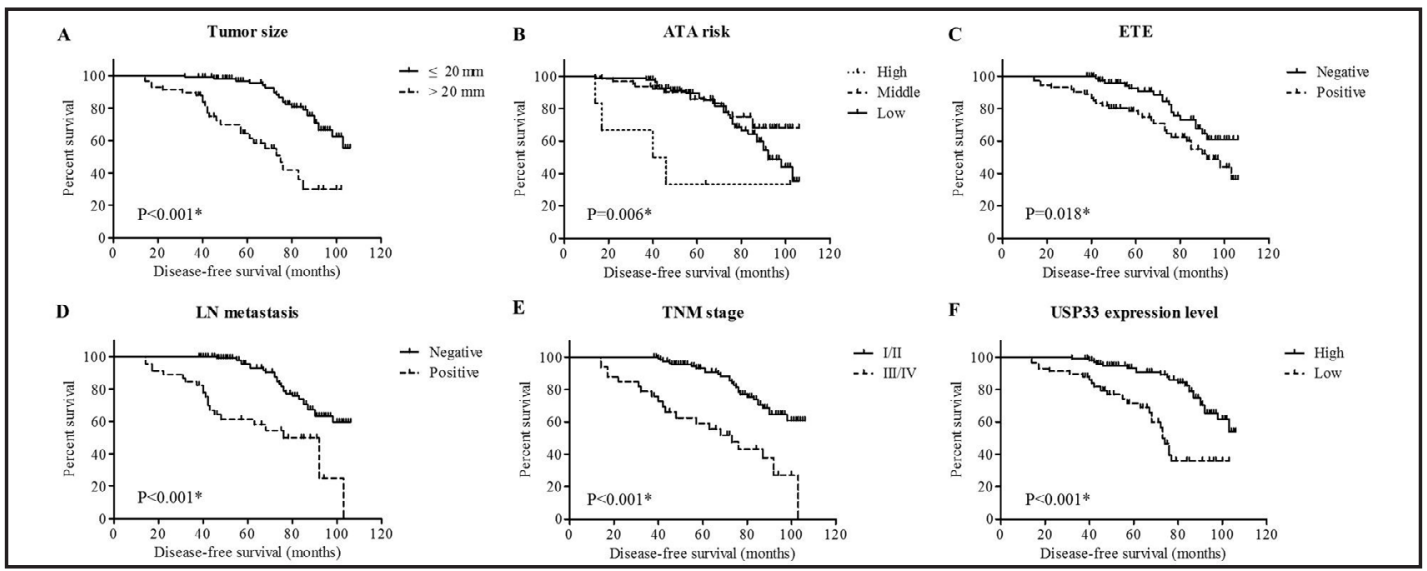

Fig. 2. USP33 expression level is helpful in predicting clinical outcomes of PTC patients. Kaplan-Meier survival analyses were performed based on all pathological characteristics and USP33 protein levels, respectively. The survival data revealed that tumor size (A), ATA risk (B), ETE status (C), LN metastasis (D), TNM stage (E), and USP33 protein level (F) were all factors that can influence the disease recurrence $(\mathrm{P}<0.05)$. 
suppressed by USP33 overexpression when compared to the mock control (Fig. 3C, 3D). On the other hand, USP33 knockdown by siRNA significantly enhanced the cell migration and invasion capacities. Collectively, cellular studies suggested the suppressive effects of USP33 on the progression of PTC.

\section{USP33 is partially function through Robo1 signaling}

Recent studies have reported that USP33 may mediate oncogenic signaling through the Slit-Robo signaling pathway in colorectal cancer and breast cancer [18-20]. To further explore the potential mechanism that might be involved in the USP33-associated malignant progression of PTC, we firstly tested the protein expression patterns of Robo1 in PTC tissues, and identified the positive correlations between Robo1 and USP33 levels (Fig. 4A, P<0.01). Next, we transfected the TPC-1 cells with HA-tagged Robo 1 and Flag-tagged USP33 plasmids, and confirmed the protein interactions between USP33 and Robo1 by co-immunoprecipitation assay (Fig. 4B). Additionally, we tested the cell viability after double-transfecting USP33 and Robo1 plasmids into TPC-1 cells, and found that co-transfection of Robo1 remarkably enhanced the tumor-suppressing effect of USP33. On the other hand, double-knockdown of Robo1 with USP33 showed an up-regulated cell viability than USP33 single-knockdown (Fig. 4C). Finally, we compared the invasion ability of PTC cells in different transfection groups, which revealed that double knockdown of USP33 and Robo1 was more potent in enhancing cell malignancy. Reversely, co-overexpression of USP33 and Robo1 significantly inhibited the cell invasion (Fig. 4D), indicating that USP33 inhibit the PTC progression at least partially through Robo1 signaling.

Table 3. Multivariate analysis. ${ }^{*} \mathrm{P}<0.05$ was considered as statistically significant. Abbreviations: ATA, American Thyroid Association; ETE, Extrathyroidal extension; LN, Lymph node; USP33, Ubiquitin specific peptidase 33

\section{Discussion}

PTC is generally curable by thyroidectomy combined with radioiodine therapy, but many patients

\begin{tabular}{lccc}
\hline Variables & Hazard ratio & 95\% Confidence Interval & P value \\
\hline Tumor size & 4.06 & $2.03-8.11$ & $<0.001^{*}$ \\
ATA risk & 0.87 & $0.48-1.58$ & 0.640 \\
ETE & 2.34 & $1.24-4.44$ & $0.009^{*}$ \\
LN metastasis & 2.19 & $1.12-4.28$ & $0.022^{*}$ \\
TNM stage & 1.91 & $1.00-3.64$ & $0.049^{*}$ \\
USP33 expression & 2.09 & $1.12-3.89$ & $0.020^{*}$ \\
\hline
\end{tabular}

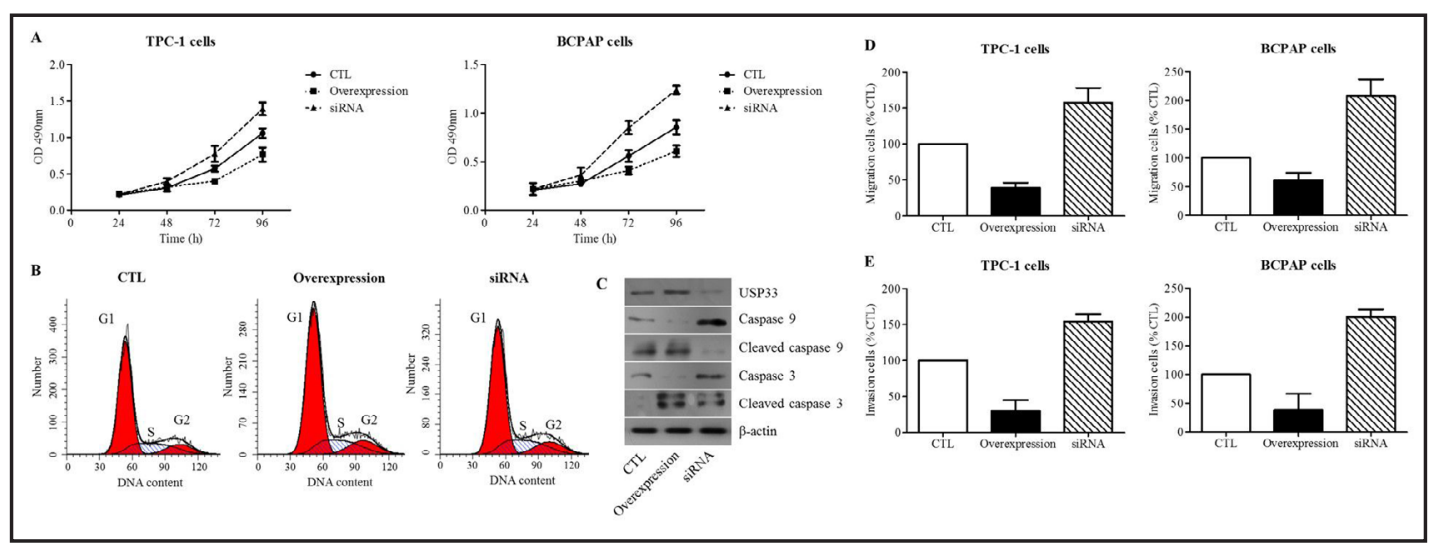

Fig. 3. USP33 attenuates the viability, migration and invasion of PTC cells. (A) MTT experiments showed USP33 overexpression attenuated cell viability in both TPC-1 and BCPAP cell lines, while USP33-siRNA enhanced cell viability. (B) Fluorescence-activated cell sorting (FACS) data showed no significant alteration in cell cycle either treated by USP33 overexpression or siRNA. (C) Western blot results revealed that USP33 can activate caspase 9 and caspase 3 proteins, indicating its possible role in promoting cell apoptosis. Wound healing assay (D) and Matrigel-Transwell assay (E) confirmed the role of USP33-overexpression in inhibiting the migration and invasion of PTC cells, whereas USP33-siRNA showed opposite effects. 
suffer disease recurrence

$[21,22]$. Therefore, specific biomarkers for risk evaluation are helpful to identify patients at high recurrence so active treatment and careful monitoring can be provided. The well-established risk classification is based on age, tumor size, histological subtypes, the status of ETE and LNM, and tumor stages. However, more and more studies revealed that even patients under the similar conditions as above, may show completely heterogeneity and uncertainty clinical outcomes. In this context, molecular biomarkers are more powerful on its role in revealing detailed functional mechanisms and directional for drug treatment.

USP33, also named VDU1 (VHL-interacting deubiquitinating enzyme 1 ), is a deubiquitinase that regulates the tumor biology of colorectal cancer, lung cancer, and breast cancer [18-20]. It was reported

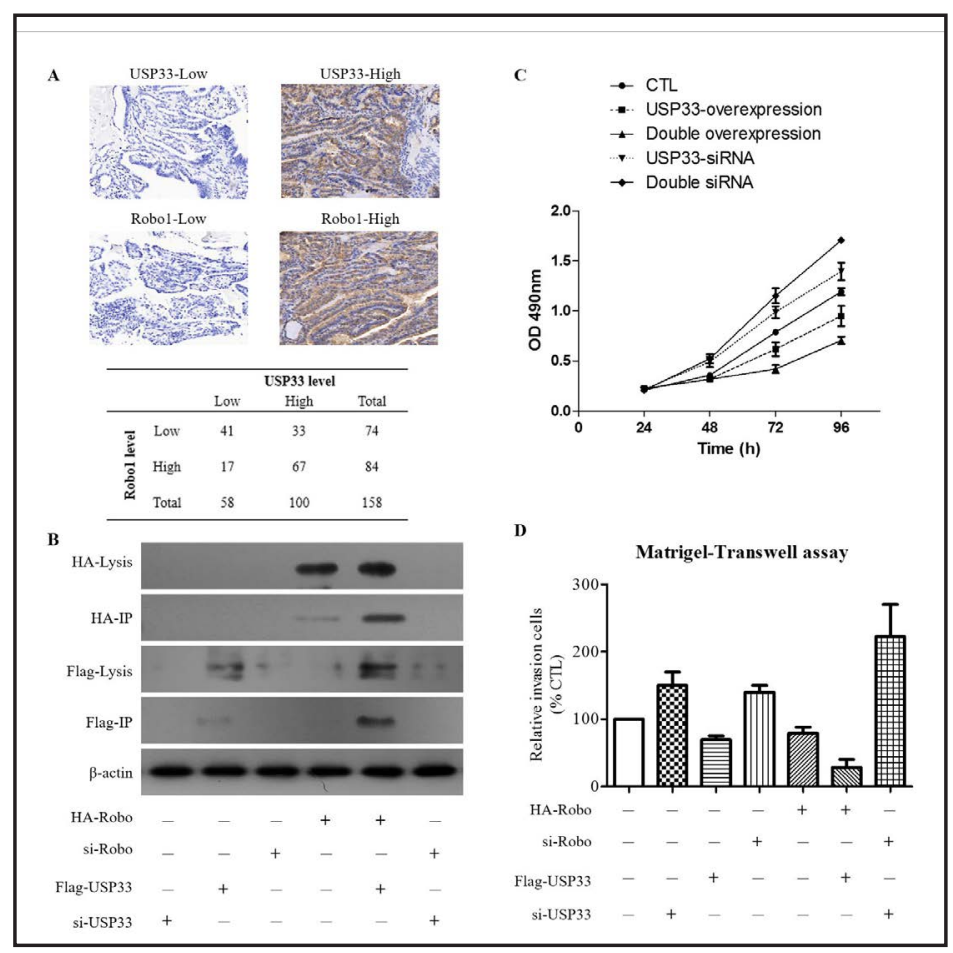

Fig. 4. Robo1 shows positively correlations with USP33 from both expression and function aspects. (A) The expression of Robo1 protein was tested by IHC, which showed a primary cytoplasm localization. In addition, the expression levels of USP33 and Robo1 was tested by chi-square test, and showed a positive correlation between these two proteins $(\mathrm{P}<0.001)$. (B) TPC-1 cells were transfected with different plasmids or siRNAs, co-immunoreaction results showed that USP33 can interact with Robo1 protein, which was consistent with the IHC data. Knockdown of USP33 increased cell viability (C) and invasion (D), and Robo1 knockdown simultaneously can further enhance its oncogenic effects. Reversely, co-overexpression of USP33 and Robo1 exhibited higher inhibition efficacy than single transfection. that USP33 expression is negatively correlated with the prognosis of certain malignancies. However, the functions and mechanisms of USP33 in PTC has remained unknown. Therefore, we investigated the expression and clinical significance of USP33 in the tumor tissues of PTC patients, which revealed an anti-tumor potential of this specific deubiquitinase. Additionally, its effects on cell proliferation, migration and invasion were tested using USP33 siRNA knockdown or overexpression in two PTC cell lines. The results showed that USP33 knockdown significantly up-regulated the viability and invasion capacity of TPC-1 and BCPAP cells. Further protein level experiments demonstrated that USP33 can bind with Robo1 protein, which maybe the underlying anti-tumor mechanism.

Robo1 is the major receptor of Slit proteins. Slit is a family of secreted extracellular matrix proteins which functions in the neural development and tumor development [23]. Slit proteins were involved in the angiogenesis and migration of tumor, normally function as tumor suppressor [24]. Absences in the expression of Slit proteins are associated with a variety of malignancies [25]. Importantly, Slit-Robo interaction has been implicated in hormone dependent cancers, particularly in females [26]. The role of Slit-Robo and its functions in cancer treatment and development are becoming increasingly unraveled but also increasingly complex. Consistent with in colorectal cancer and breast cancer $[18,19]$, our results showed that USP33 can function by interacting with Robo1 in PTC cells, which 
may enhancing the Slit-Robo signaling and thus inhibit tumor progression. Furthermore, our data suggested that USP33 can activate caspase 9 and caspase 3 proteins, in accordance with the fact that Robo1 functions partly by inducing cell apoptosis [26]. Taking into consideration that USP33 is an enzyme catalyzing deubiquitinate process, the interaction with Robo1 may modulate its ubiquitinating status. Indeed, several studies have reported that the ubiquitination of Robo1 was negatively regulated by USP33 [20, 27]. However, we cannot ignore other potential effects by USP33-Robo1 interaction. For example, USP33 can indirectly bind with srGAP1 (Slit-Robo Rho GTPase activating protein 1) with the assistance of Robo1, subsequently exerting anti-migration effects in colorectal cancer cells [28].

Besides Slit-Robo1 signaling pathways, USP33 can also regulate the signaling of C-X-C chemokine receptor type 4 (CXCR4) by deubiquitinate beta-arrestin as reported by Liu. et al [29].. That's interesting because CXCR4-beta-arrestin interaction can regulates its downstream ERK signaling. ERK signaling plays central roles in the carcinogenesis and progression of common cancers, its dysregulated expression or phosphorylation also affect the levels of its potential downstream targets, which are responsible for a wide range of biological processes including cell proliferation and differentiation, migration and invasion, etc [30]. Therefore, it's also possible that USP33 may also function in PTC partially through CXCR4-beta-arrestin signaling pathway, which need further experimental verification.

\section{Conclusion}

Taken together, our study indicated that USP33 downregulation was correlated with higher recurrence risk of PTC patients. USP33 may promote PTC cell apoptosis and suppress tumor invasion through enhancing Slit-Robo1 signaling.

\section{Abbreviations}

PTC (Papillary thyroid carcinoma); PCR (Polymerase chain reaction); RT-qPCR (Quantitative real-time polymerase chain reaction); RNA (Ribonucleic acid); TNM (Tumor, nodes, metastasis); ATA (American Thyroid Association); HR (Hazard ratio); CI (Confidence interval); mm (millimeter); USP33 (Ubiquitin specific peptidase 33); DFS (Disease-free survival); PTM (post-translational modification); DUB (Deubiquitinating enzymes); IHC (Immunohistochemistry)

\section{Acknowledgements}

This study was supported by the University Key Scientific Research Project Funding of Henan Province in 2017 [No. 17A320030].

\section{Disclosure Statement}

No conflict of interests exists.

\section{References}

1 Jemal A, Siegel R, Xu J, Ward E: Cancer statistics, 2010 CA Cancer J Clin 2010;60:277-300.

$\checkmark 2$ Guerrero MA, Clark OH: Controversies in the management of papillary thyroid cancer revisited. ISRN oncology 2011;2011

-3 Sipos JA, Mazzaferri EL: Thyroid cancer epidemiology and prognostic variables. Clin Oncol (R Coll Radiol) 2010;22:395-404.

4 Cooper D, Doherty G, Haugen B, Kloos R, Lee S, Mandel S, Mazzaferri E, McIver B, Sherman S: Tuttle rm. American thyroid association guidelines taskforce. Management guidelines for patients with thyroid nodules and differentiated thyroid cancer. Thyroid 2006;16:109-142. 


\section{Cellular Physiology Cell Physiol Biochem 2018;45:2044-2053

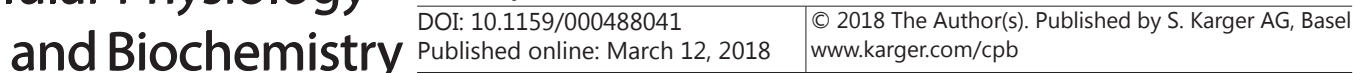

5 Kim WW, Yang SI, Kim JH, Choi YS, Park Y-H, Kwon SK: Experience and analysis of delphian lymph node metastasis in patients with papillary thyroid carcinoma. World J Surg Oncol 2012;10:226.

6 Yuan MH, Zhou RS, She B, Xu HF, Wang JY, Wei LX: Expression and clinical significance of stip1 in papillary thyroid carcinoma. Tumour Biol 2014;35:2391-2395.

7 Grant CS: Recurrence of papillary thyroid cancer after optimized surgery. Gland Surg 2015;4:52.

-8 Bode AM, Dong Z: Post-translational modification of p53 in tumorigenesis. Nat Rev Cancer 2004;4:793.

9 Dai C, Gu W: P53 post-translational modification: Deregulated in tumorigenesis. Trends Mol Med 2010;16:528-536.

10 Fraile J, Quesada V, Rodríguez D, Freije J, López-otín C: Deubiquitinases in cancer: New functions and therapeutic options. Oncogene 2012;31:2373.

-11 D’Arcy P, Linder S: Proteasome deubiquitinases as novel targets for cancer therapy. Int J Biochem Cell Biol 2012;44:1729-1738.

12 D’arcy P, Wang X, Linder S: Deubiquitinase inhibition as a cancer therapeutic strategy. Pharmacol Ther 2015;147:32-54.

13 Wilkinson KD: Regulation of ubiquitin-dependent processes by deubiquitinating enzymes. FASEB J 1997;11:1245-1256.

14 Reyes-Turcu FE, Ventii KH, Wilkinson KD: Regulation and cellular roles of ubiquitin-specific deubiquitinating enzymes. Annu Rev Biochem 2009;78:363-397.

15 Qiu W, Yang Z, Fan Y, Zheng Q: Znrf3 is downregulated in papillary thyroid carcinoma and suppresses the proliferation and invasion of papillary thyroid cancer cells. Tumour Biol 2016;37:12665-12672.

16 Zhao H, Tang H, Huang Q, Qiu B, Liu X, Fan D, Gong L, Guo H, Chen C, Lei S, Yang L, Lu J, Bao G: Mir-101 targets usp22 to inhibit the tumorigenesis of papillary thyroid carcinoma. Am J Cancer Res 2016;6:25752586.

17 Cooper DS, Doherty GM, Haugen BR, Kloos RT, Lee SL, Mandel SJ, Mazzaferri EL, McIver B, Pacini F, Schlumberger M, Sherman SI, Steward DL, Tuttle RM: Revised american thyroid association management guidelines for patients with thyroid nodules and differentiated thyroid cancer. Thyroid 2009;19:11671214.

18 Yuasa-Kawada J, Kinoshita-Kawada M, Rao Y, Wu JY: Deubiquitinating enzyme usp33/vdu1 is required for slit signaling in inhibiting breast cancer cell migration. Proc Natl Acad Sci U S A 2009;106:14530-14535.

19 Huang Z, Wen P, Kong R, Cheng H, Zhang B, Quan C, Bian Z, Chen M, Zhang Z, Chen X, Du X, Liu J, Zhu L, Fushimi K, Hua D, Wu JY: Usp33 mediates slit-robo signaling in inhibiting colorectal cancer cell migration. Int J Cancer 2015;136:1792-1802.

20 Wen P, Kong R, Liu J, Zhu L, Chen X, Li X, Nie Y, Wu K, Wu JY: Usp33, a new player in lung cancer, mediates slit-robo signaling. Protein Cell 2014;5:704-713.

-21 Schlumberger MJ: Papillary and follicular thyroid carcinoma. N Engl J Med 1998;338:297-306.

22 Ma B, Liao T, Wen D, Dong C, Zhou L, Yang S, Wang Y, Ji Q: Long intergenic non-coding rna 271 is predictive of a poorer prognosis of papillary thyroid cancer. Sci Rep 2016;6:36973.

-23 Hohenester E: Structural insight into slit-robo signalling. Biochem Soc Trans 2008;36:251-256.

24 Prasad A, Paruchuri V, Preet A, Latif F, Ganju RK: Slit-2 induces a tumor-suppressive effect by regulating $\beta$-catenin in breast cancer cells. J Biol Chem 2008;283:26624-26633.

25 Prasad A, Fernandis AZ, Rao Y, Ganju RK: Slit protein-mediated inhibition of cxcr4-induced chemotactic and chemoinvasive signaling pathways in breast cancer cells. J Biol Chem 2004;279:9115-9124.

-26 Dickinson RE, Duncan WC: The slit-robo pathway: A regulator of cell function with implications for the reproductive system. Reproduction 2010;139:697-704.

27 Yuasa-Kawada J, Kinoshita-Kawada M, Wu G, Rao Y, Wu JY: Midline crossing and slit responsiveness of commissural axons require usp33 Nat Neurosci 2009;12:1087.

28 Feng Y, Feng L, Yu D, Zou J, Huang Z: Srgap1 mediates the migration inhibition effect of slit2-robo1 in colorectal cancer. J Exp Clin Cancer Res 2016;35:191.

29 Liu H, Zhang Q, Li K, Gong Z, Liu Z, Xu Y, Swaney MH, Xiao K, Chen Y: Prognostic significance of usp33 in advanced colorectal cancer patients: New insights into beta-arrestin-dependent erk signaling. Oncotarget 2016;7:81223-81240.

-30 Wang F, Ying H-Q, He B-S, Pan Y-Q, Deng Q-W, Sun H-L, Chen J, Liu X, Wang S-K: Upregulated lncrna-uca1 contributes to progression of hepatocellular carcinoma through inhibition of mir-216b and activation of fgfr1/erk signaling pathway. Oncotarget 2015;6:7899. 